\title{
Shape Analysis for Ultrasound Breast Lesion Evaluation
}

\author{
Miguel Alemán-Flores ${ }^{1}$, Patricia Alemán-Flores ${ }^{2}$, Luis Álvarez-Leén ${ }^{1}$, \\ Rafael Fuentes-Pavón ${ }^{2}$, José M. Santana-Montesdeoca ${ }^{2}$ \\ ${ }^{1}$ Departamento de Informática y Sistemas \\ Universidad de Las Palmas de Gran Canaria, 35017, Las Palmas, Spain \\ ${ }^{2}$ Sección de Ecografía, Servicio de Radiodiagnóstico \\ Hospital Universitario Insular de Gran Canaria, 35016, Las Palmas, Spain \\ Email: maleman@dis.ulpgc.es
}

\begin{abstract}
This work presents a new approach for a wide analysis of the shape of breast tumors in ultrasound images. We have developed an environment for the filtering of the images, the segmentation of the nodules and the extraction of a series of measurements which describe the shape of the nodules. This will help in the computer-aided evaluation of the tumors and the distinction of benign and malignant nodules. The results extracted for the different features are coherent with the assessment of the specialists and represent a great help for the examination of the images and the decision making process.
\end{abstract}

\section{Introduction}

Ultrasonography is widely used for the detection and evaluation of many diseases. In the case of breast cancer, it is a very useful complementary imaging technique to mammography. Not only does it provide a different assessment of the lesion, but it also allows detecting very small lesions and analyzing dense breasts, which is quite difficult using mammography. Furthermore, a series of features related to the shape of the nodule, the regularity of the contour and the intensity and contrast between different regions have been described to characterize the lesions and help distinguish benign from malignant nodules [1]. In this work, we present a common framework which, by means of several computer vision and image processing techniques, allows extracting some measurements of the shape of a nodule. These include the roundness of its shape, the size of ramifications, the number of microlobulations and angular margins, the presence of spiculation or a taller-than-wide shape.

In order to accelerate and simplify this task, a semi-automatic segmentation of the lesion is performed, so that it is no necessary to delineate the lesion manually. This is carried out by filtering the image using a speckle noise reducing filter, obtaining a presegmentation using a region-growing algorithm, and refining the presegmentation using active contours.

Due to the difficulties that speckle characteristic noise represents for the automatic analysis of ultrasound images, little work has been done in this filed, 
and the results are usually moderate. Some previous works try to adjust the parameters of the ultrasound systems to help in the decision making process [2], segment the tissues [3], or deal with certain particular aspects of the nodules, such as their texture [4]. Other works deal with the detection of the lesions [5] or a general description of the nodules [6]. However, we intend to obtain a more detailed description of the shape of the nodules, so that their benignity or malignancy can be determined in a more accurate way.

\section{Methods}

In this section, we present the methods we have used for the segmentation of the nodules and for the shape analysis, according to the diagnostic criteria described by the specialists.

\subsection{Filtering and segmentation}

Before analyzing the shape of the tumor, it must be segmented from the surrounding tissues. Due to the characteristic speckle noise of ultrasound images, a noise reducing filter must first be applied. We use the truncated median filter, which, in a few iterations, progressively reduces the speckles and provides quite satisfactory results [7]. From an inner point, which is the only interaction asked to the physician, a region-growing algorithm is used to extract an initial presegmentation. This technique is very fast, but does not provide accurate enough results. However, it can be used to obtain an initial contour for the active contour technique [8][9], which generates very precise segmentations if it is initialized with a close approximation. For the implementation we apply a level set approach [10]. Once the segmentation is obtained, we proceed to analyze the shape of the nodule.

\subsection{Shape analysis}

The main features which allow discriminating benign from malignant tumors, thus avoiding performing the biopsy, are related to the shape of the nodules and the regularity of their contours. When analyzing the general shape of the tumor, an ellipsoid shape or the presence of a few gentle and well circumscribed lobulations are considered as benignity findings. On the other hand, ramifications or a taller-than-wide shape are considered as malignancy findings. Moreover, when examining the smoothness of the contour, if it presents microlobulations, angular margins or spiculation, they are interpreted as malignancy findings, as opposite to a rounded and well defined contour. In this work, we analyze both, the global and the local features of the contours, so that some robust, reproducible and accurate measurements can be provided to the specialists in order to perform a more reliable distinction between benign and malignant nodules.

The ideal benign nodule has a well defined ellipsoid shape. We not only intend to binary decide whether a nodule is ellipsoidal or not, but we also try to measure 
how ellipsoidal it is. In order to automatically determine it, we extract the ellipse which best fits the contour of the nodule. We apply a gradient descent technique in which the five parameters of the ellipse (two coordinates for the center, two dimensions for the axes and an angle for the orientation) are iteratively adjusted. Since we need some initial values, we roughly estimate them from the position, the dimensions and the orientation of the segmentation. The distance from the contour to the final ellipse, with respect to the size of the nodule, measures how ellipsoidal the lesion is. If it is not enough, we search for two or three gentle lobulations by splitting the contour at the closest points to the center.

Once the minimum distance ellipse (or ellipses) has been found, the regions where it clearly differs from the contour allow locating the eventual ramifications of the nodule. Their relative size with respect to the total size indicates their relevance. To decide whether a nodule is likely to invade different tissues, we consider the ratio between its dimensions, since the different tissue layers are supposed to be approximately horizontal.

The local variations of the contour require a detailed analysis of its regularity. Angular margins are identified by searching for pseudo-corners on the contour of the nodule. Microlobulations are extracted using a similar approach to that used for ellipse extraction, but, in this case, we search for small arcs of ellipses which fit certain parts of the contour. Spiculation is measured through the variance of the orientation of the gradient along the contour, for which the structure tensor is used.

\section{Results}

Figure 1 shows some examples of nodules which have been segmented on the ultrasound images. As observed, the segmentations are quite satisfactory and allow a precise analysis of the shape of the nodules.

Table 1 shows the values of the different measurements for the set of nodules shown in figure 1. For every feature, we show the numerical measurement provided by the system (sys), as well as the assessment performed by three specialists (phy). As observed, not only is it coherent with the appearance of the nodule, but it also allows setting a threshold for each feature if the values are compared with the binary assessment of the physicians. Only isolated cases differ and, in most cases, they are due to a reduction of the specificity produced by trying to adjust the parameters and thresholds to increase the sensitivity, since it is better to perform a biopsy of a benign nodule than overlooking a malignant one.

\section{Discussion}

In this paper we have presented a common framework for the assessment of a set of parameters regarding the shape of a breast lesion in an ultrasound image. These measurements allow analyzing the shape of the nodule in a robust and accurate way and provide numerical values which describe the contour in terms 
Fig. 1. Examples of breast nodules in ultrasound images and their corresponding automatic segmentations

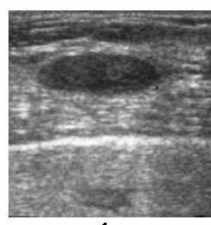

1
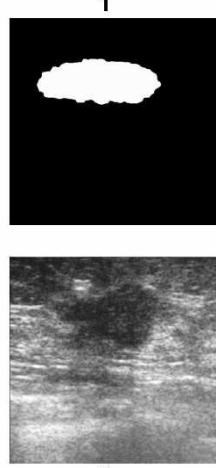

6

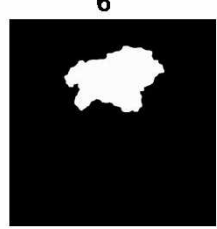

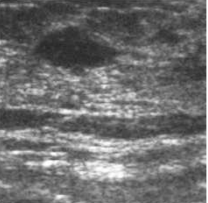

2
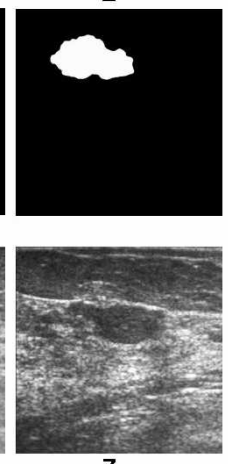

7

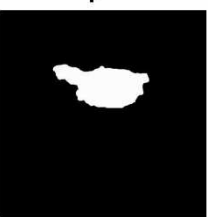

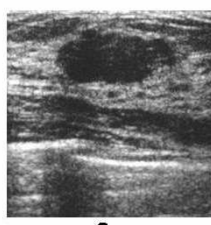

3
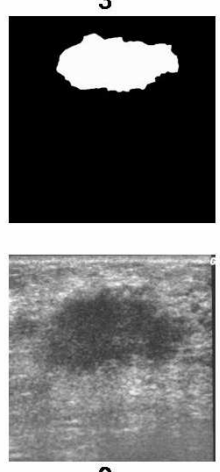

8

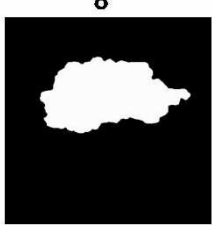

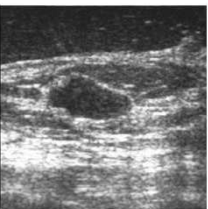

4
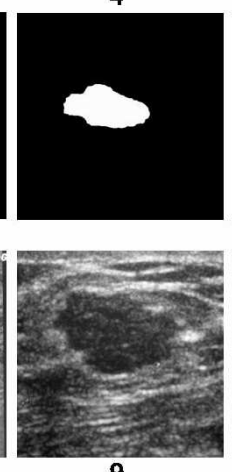

9

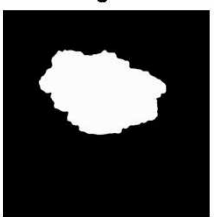

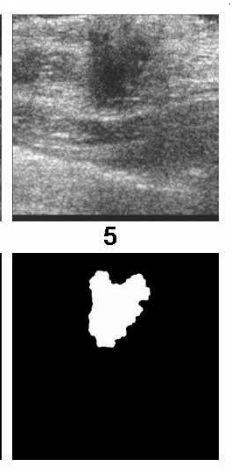
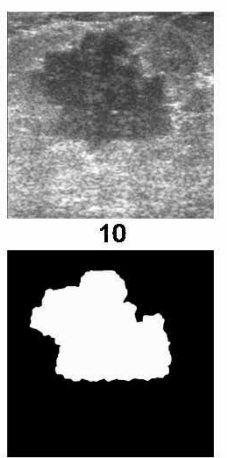

Table 1. Numerical measurements obtained by the system (sys) and assessment of the specialist physicians (phy) for the nodules in fig. 1 regarding the features related to the shape of the nodules: ellipsoid shape (ES), gentle lobulations (GL), ramifications $(\mathrm{RM})$, taller-than-wide shape (TW), angular margins (AM), microlobulations (ML) and spiculation (SP). In the values of the specialists, 0 means that a malignant factor has not been found or a benignant finding has been found, and 1 means the opposite

\begin{tabular}{c|cc|cc|cc|cc|cc|cc|cc}
\hline & \multicolumn{2}{|c|}{ ES } & \multicolumn{2}{c|}{ GL } & \multicolumn{2}{c|}{ RM } & \multicolumn{2}{c|}{ TW } & \multicolumn{2}{c|}{ AM } & \multicolumn{2}{c|}{ ML } & \multicolumn{2}{c}{ SP } \\
Nodule & sys & phy & sys & phy & sys & phy & sys & phy & sys & phy & sys & phy & sys & phy \\
\hline 1 & 1.9 & 0 & 2.2 & 0 & 0.0 & 0 & 0.36 & 0 & 3.9 & 0 & 1.8 & 0 & 0.8 & 0 \\
2 & 6.1 & 1 & 3.9 & 0 & 7.6 & 0 & 0.53 & 0 & 5.1 & 1 & 2.4 & 0 & 0.0 & 0 \\
3 & 3.4 & 0 & 5.2 & 1 & 5.7 & 0 & 0.47 & 0 & 5.2 & 1 & 1.8 & 0 & 1.7 & 0 \\
4 & 7.3 & 1 & 3.2 & 0 & 9.4 & 0 & 0.50 & 0 & 3.3 & 0 & 0.6 & 0 & 0.9 & 0 \\
5 & 10.7 & 1 & 10.5 & 1 & 13.4 & 1 & 1.24 & 1 & 6.5 & 1 & 3.1 & 1 & 0.0 & 0 \\
6 & 10.3 & 1 & 9.4 & 1 & 12.5 & 1 & 0.65 & 0 & 4.8 & 1 & 3.7 & 1 & 1.4 & 0 \\
7 & 7.0 & 1 & 12.4 & 1 & 13.6 & 1 & 0.45 & 0 & 4.3 & 1 & 3.1 & 1 & 1.2 & 0 \\
8 & 10.6 & 1 & 12.5 & 1 & 7.1 & 0 & 0.66 & 0 & 5.0 & 1 & 3.1 & 1 & 0.8 & 0 \\
9 & 7.0 & 1 & 8.9 & 0 & 4.7 & 0 & 0.50 & 0 & 5.5 & 1 & 2.4 & 1 & 2.5 & 1 \\
10 & 19.9 & 1 & 12.4 & 1 & 8.7 & 0 & 0.78 & 0 & 5.0 & 1 & 6.8 & 1 & 1.4 & 0 \\
\hline
\end{tabular}


of global and local regularity. Prior to the analysis of the shape, the segmentation, performed using a noise reduction filter, a region growing presegmentation and active contours, provides quite satisfactory results, while it simplifies and accelerates the process of delimiting the nodules.

The numerical techniques we have applied allow examining global features, such as how ellipsoidal a nodule is, whether it has two or three gentle lobulations and whether it has ramifications. On the other hand, the local variations in the contour help determine whether it has angular margins, microlobulations or spiculation.

The measurements are coherent with the assessment of the specialists and, instead of providing a binary evaluation, they generate scalar values which permit determining in what degree each factor is present and what the probability is of being a benign or malignant tumor. Our results confirm the usefulness of image processing techniques in the evaluation of ultrasound for a precise early detection of breast cancer.

\section{Acknowledgements}

This work was partially supported by project TIN 2005 02004, Ministerio de Educación y Ciencia, Spain.

\section{References}

1. Stavros AT, Thickman D, Rapp CL, et al. Solid breast nodules: Use of sonography to distinguish between benign and malignant lesions. Radiology 1995;196(1):123134.

2. Kuo WJ, Chang RF, Moon WK, et al. Computer-aided diagnosis of breast tumors with different US systems. Acad Radiol 2002;9(1):793-799.

3. Kaufhold J, Chan R, Karl WC, Castanon DA. Ultrasound tissue analysis and characterization. Procs of SPIE 1999; 73-83.

4. Chen DR, Chang RF, Juang YL. Computer-aided diagnosis applied to US of solid breast nodules by using neural networks. Radiology 1999;213(1):407-412.

5. Drukker K, Giger ML, et al KHorsch. Computerized lesion detection on breast ultrasound. Medical Physics 2002;29(7):1438-1446.

6. Revell J, Mirmehdi M, McNally D. Applied review of ultrasound image feature extraction methods. In: Procs. of The 6th Medical Image Understanding and Analysis Conference; 2002. 173-176.

7. Davis ER. On the noise suppression and image enhancement characteristics of the median, truncated median and mode filters. Pattern Recognition Letters 1988;7:87-97.

8. Caselles V, Kimmel R, Sapiro G. Geodesic active contours. International Journal of Computer Vision 1997;22(1):61-79.

9. Kass M, Witkin A, Terzopoulos D. Active Contour Models. In: Procs. of 1st International Conference on Computer Vision; 1987. 259-268.

10. Osher S, Sethian J. Fronts propagating with curvature dependent speed: algorithms based on the Hamilton-Jacobi formulation. Journal of Computational Physics 1988;79:12-49. 